Anna Anop, Tetiana Kasirenko, and Aleksandr Murach

(Institute of Mathematics, National Academy of Sciences of Ukraine, Kyiv)

\title{
NONREGULAR ELLIPTIC BOUNDARY-VALUE PROBLEMS AND HÖRMANDER SPACES
}

\author{
Анна Аноп, Тетяна Касіренко і Олександр Мурач \\ (Інститут математики НАН України, Київ)

\section{НЕРЕГУЛЯРНІ ЕЛІПТИЧНІ КРАЙОВІ ЗАДАЧІ ТА ПРОСТОРИ ХЕРМАНДЕРА}

We investigate nonregular elliptic problems with boundary conditions of higher orders. We prove that these problems are Fredholm on appropriate pairs of inner product Hörmander spaces that form a two-sided refined Sobolev scale. We also prove a theorem on the regularity of generalized solutions to the problems in these spaces.

Досліджено нерегулярні еліптичні задачі з крайовими операторами вищих порядків. Доведено, що ці задачі є нетеровими у відповідних парах гільбертових просторів Хермандера, які утворюють двобічну уточнену соболєвську шкалу. Доведено також теорему про регулярність узагальнених розв'язків досліджуваних задач у цих просторах.

1. Вступ. Ця робота присвячена дослідженню в класах просторів Хермандера еліптичних задач, для яких максимум порядків крайових операторів більший за порядок еліптичного рівняння, або рівний йому. Такі задачі є нерегулярними еліптичними; для них не виконується класична формула Гріна, що ускладнюе їх дослідження. Змістовні приклади цих задач зустрічаються в акустиці, гідродинаміці, теорії випадкових процесів [1-3].

Зазначені задачі досить повно досліджено у двобічній шкалі просторів Соболєва, модифікованих за Ройтбергом (див. монографії [4] (розд. 4, 7) і [5] (п. 4.1)). Доведено теореми про нетеровість цих задач і регулярність їх розв'язків у просторах СоболєваРойтберга. Останні збігаються з соболєвськими просторами, якщо їх порядок регулярності є достатньо великим числом; у противному разі вони містять елементи, які не є розподілами. Втім, соболєвська шкала, градуйована за допомогою числового показника регулярності, є занадто грубою для низки задач теорії диференціальних рівнянь і математичного аналізу. На це вказував Л. Хермандер [6,7] ще у 1963 р., який увів і дослідив широкі класи нормованих просторів, для яких показником регулярності служить досить загальна функція, та застосував їх до дослідження рівнянь з частинними похідними. В останні двадцять років простори Хермандера та їх різні узагальнення широко застосовуються у різних розділах математики [8-13].

Недавно В. А. Михайлець і О. О. Мурач [14-19] побудували загальну теорію розв'язності еліптичних крайових задач у гільбертових просторах Хермандера $H^{s, \varphi}$, які утворюють уточнену соболєвську шкалу (їх результати підсумовано в $[9,20]$ ). Показниками регулярності для цих просторів служать дійсне число $s$ і функція $\varphi:(0, \infty) \rightarrow(0, \infty)$, повільно змінна на нескінченності за Караматою. Функціональний параметр $\varphi$ уточнює основну регулярність $s$. У випадку $\varphi(\cdot) \equiv 1$ простір $H^{s, \varphi}$ стає гільбертовим простором Соболєва $H^{s}$ порядку $s$. Зовсім недавно ця теорія була доповнена у статтях [21-23], де застосовано більш широкі класи гільбертових просторів Хермандера [24,25]. Відмітимо також роботу [26], в якій досліджено еліптичні з параметром крайові задачі у нормованих просторах з функціональним показником регулярності. 
Проте, еліптичні задачі, яким присвячено цю роботу, не були охоплені згаданою теорією. Мета нашої роботи - довести теореми про нетеровість досліджуваних задач і регулярність їх узагальнених розв'язків у двобічній уточненій соболєвській шкалі. У випадку, коли числовий показник $s>m+1 / 2$, де $m-$ максимум порядків крайових операторів, відповідні версії цих теорем доведено нами в [27]. Випадок $s \leq m+1 / 2$ істотно більш складний для дослідження, оскільки у ньому ліві частини крайових умов не можна коректно означити на класі $H^{s, \varphi}(\Omega)$ ров'язків еліптичного рівняння, заданого в обмеженій евклідовій області $\Omega$ з гладкою межею. На відміну від згаданих монографій $[4,5]$, ми дотримуємося в ідейному плані підходу Ж.-Л. Ліонса і Е. Мадженеса [28-30], розробленому для регулярних еліптичних крайових задач у двобічній соболєвській шкалі. Ми обмежуємося розглядом розв'язків $u \in H^{s, \varphi}(\Omega)$ еліптичного рівняння, права частина якого достатньо регулярна - належить простору Соболєва $H^{\lambda}(\Omega)$, де $\lambda>m+1 / 2-2 q$, а $2 q-$ порядок цього рівняння. Як буде показано, крайові умови допускають коректне означення на класі усіх таких розв'язків, а відповідна еліптична крайова задача володіє властивостями, подібними до її властивостей у випадку $s>m+1 / 2$. Наскільки нам відомо, отримані у цій роботі результати $є$ новими і для соболєвських просторів.

Зауважимо, що у випадку, коли порядки крайових умов менші за порядок еліптичного рівняння, різні версії теорем Ліонса - Мадженеса про нетеровість еліптичних крайових задач доведено в [31-33] для просторів Соболєва і в [34,35] для уточненої соболєвської шкали (частина цих результатів викладена у монографії [9] (пп. 4.4, 4.5)).

2. Постановка задачі. Нехай $\Omega$ - довільна обмежена область в $\mathbb{R}^{n}$, де ціле $n \geq 2$. Припускаємо, що їі межа $\Gamma$ є нескінченно гладким компактним многовидом вимірності $n-1$, причому $C^{\infty}$-структура на $\Gamma$ породжена простором $\mathbb{R}^{n}$.

Розглянемо в області $\Omega$ таку крайову задачу:

$$
\begin{gathered}
A u=f \quad \text { в } \quad \Omega, \\
B_{j} u=g_{j} \quad \text { на } \Gamma, \quad j=1, \ldots, q .
\end{gathered}
$$

Тут $A:=A(x, D)$ - лінійний диференціальний оператор на $\bar{\Omega}:=\Omega \cup \Gamma$ довільного парного порядку $2 q \geq 2$, а кожне $B_{j}:=B_{j}(x, D)$ - крайовий лінійний диференціальний оператор на $\Gamma$ довільного порядку $m_{j} \geq 0$. Усі коефіцієнти цих диференціальних операторів є нескінченно гладкими комплекснозначними функціями, заданими на $\bar{\Omega} \mathrm{i}$ Г відповідно. Узагалі в роботі розподіли та функції вважаємо комплекснозначними і тому розглядаємо комплексні функціональні простори.

Припускаємо, що крайова задача $(1),(2)$ еліптична в області $\Omega$, тобто диференціальний оператор $A$ є правильно еліптичним на $\bar{\Omega}$, а набір $B:=\left(B_{1}, \ldots, B_{q}\right)$ крайових диференціальних операторів задовольняє умову Лопатинського щодо $A$ на $\Gamma$ (див., наприклад, огляд [36] (п. 1.2) або довідник [37] (розд. III, § 6, пп. 1, 2)). Окрім того, припускаємо, що

$$
m:=\max \left\{m_{1}, \ldots, m_{q}\right\} \geq 2 q .
$$

Отже, еліптична крайова задача (1), (2) є нерегулярною. Задля більшої лаконічності формул покладемо $r:=m+1$.

Пов'яжемо із цією задачею лінійне відображення

$$
u \mapsto(A u, B u)=\left(A u, B_{1} u, \ldots, B_{q} u\right), \quad \text { де } \quad u \in C^{\infty}(\bar{\Omega}) .
$$

Будемо досліджувати властивості продовження за неперервністю цього відображення у підходящих парах функціональних просторів Хермандера. 
Для опису області значень цього продовження нам знадобиться така спеціальна формула Гріна [5] (формула (4.1.10)):

$$
\begin{gathered}
(A u, v)_{\Omega}+\sum_{j=1}^{r-2 q}\left(D_{\nu}^{j-1} A u, w_{j}\right)_{\Gamma}+\sum_{j=1}^{q}\left(B_{j} u, h_{j}\right)_{\Gamma}= \\
=\left(u, A^{+} v\right)_{\Omega}+\sum_{k=1}^{r}\left(D_{\nu}^{k-1} u, K_{k} v+\sum_{j=1}^{r-2 q} R_{j, k}^{+} w_{j}+\sum_{j=1}^{q} Q_{j, k}^{+} h_{j}\right)_{\Gamma}
\end{gathered}
$$

для довільних $u, v \in C^{\infty}(\bar{\Omega})$ i $w_{1}, \ldots, w_{r-2 q}, h_{1}, \ldots, h_{q} \in C^{\infty}(\Gamma)$. Тут і далі через $(\cdot, \cdot)_{\Omega}$ i $(\cdot, \cdot)_{\Gamma}$ позначено скалярні добутки у гільбертових просторах $L_{2}(\Omega)$ і $L_{2}(\Gamma)$ функцій квадратично інтегровних відповідно на $\Omega$ і $\Gamma$ за мірою Лебега, а також продовження за неперервністю цих скалярних добутків. Окрім того, $D_{\nu}:=i \partial / \partial \nu$, де $i-$ уявна одиниця, а $\nu$ - поле ортів внутрішніх нормалей до межі Г. Як звичайно, $A^{+}-$диференціальний оператор, формально спряжений до $A$ відносно $(\cdot, \cdot)_{\Omega}$. Окрім того, всі $R_{j, k}^{+}$і $Q_{j, k}^{+}$є дотичними диференціальними операторами, формально спряженими відповідно до $R_{j, k} \mathrm{i}$ $Q_{j, k}$ відносно $(\cdot, \cdot)_{\Gamma}$. Тут дотичні лінійні диференціальні оператори $R_{j, k}:=R_{j, k}\left(x, D_{\tau}\right)$ i $Q_{j, k}:=Q_{j, k}\left(x, D_{\tau}\right)$ узяті із зображення крайових диференціальних операторів $D_{\nu}^{j-1} A$ i $B_{j}$ у вигляді

$$
\begin{aligned}
D_{\nu}^{j-1} A(x, D) & =\sum_{k=1}^{r} R_{j, k}\left(x, D_{\tau}\right) D_{\nu}^{k-1}, \quad j=1, \ldots, r-2 q, \\
B_{j}(x, D) & =\sum_{k=1}^{r} Q_{j, k}\left(x, D_{\tau}\right) D_{\nu}^{k-1}, \quad j=1, \ldots, q .
\end{aligned}
$$

Зауважимо, що $\operatorname{ord} R_{j, k} \leq 2 q+j-k$ i ord $Q_{j, k} \leq m_{j}-k+1$, причому $R_{j, k}=0$ при $k \geq 2 q+j+1$ і $Q_{j, k}=0$ при $k \geq m_{j}+2$. Нарешті, кожне $K_{k}:=K_{k}(x, D)$ є деяким крайовим лінійним диференціальним оператором на $Г$ порядку ord $K_{k} \leq 2 q-k$ з коефіцієнтами класу $C^{\infty}(\bar{\Omega})$; при цьому $K_{k}=0$, якщо $k \geq 2 q+1$.

Беручи до уваги спеціальну формулу Гріна, розглянемо таку крайову задачу в області $\Omega$ з $r-q$ додатковими невідомими функціями на межі $\Gamma$ :

$$
\begin{gathered}
A^{+} v=\omega \quad \text { в } \Omega, \\
K_{k} v+\sum_{j=1}^{r-2 q} R_{j, k}^{+} w_{j}+\sum_{j=1}^{q} Q_{j, k}^{+} h_{j}=\psi_{k} \quad \text { на } \quad \Gamma, \quad k=1, \ldots, r .
\end{gathered}
$$

Тут функція $v$ на $\Omega$ і $r-q$ функцій $w_{1}, \ldots, w_{r-2 q}, h_{1}, \ldots, h_{q}$ на $\Gamma$ є невідомими. Ця задача називається формально спряженою до задачі (1), (2) відносно розглянутої спеціальної формули Гріна. Як відомо [5] (теорема 4.1.1), крайова задача (1), (2) еліптична в області $\Omega$ тоді і лише тоді, коли формально спряжена задача (4), (5) еліптична в $\Omega$ як крайова задача з додатковими невідомими функціями на межі області.

Позначимо через $N$ лінійний простір усіх розв'язків $u \in C^{\infty}(\bar{\Omega})$ крайової задачі $(1)$, (2) у випадку, коли $f=0$ в $\Omega$ і кожне $g_{j}=0$ на $Г$. Окрім того, позначимо через $N_{\star}$ лінійний простір усіх розв'язків

$$
\left(v, w_{1}, \ldots, w_{r-2 q}, h_{1}, \ldots, h_{q}\right) \in C^{\infty}(\bar{\Omega}) \times\left(C^{\infty}(\Gamma)\right)^{r-q}
$$

формально спряженої крайової задачі (4), (5) у випадку, коли $\omega=0$ в $\Omega$ і кожне $\psi_{k}=0$ на Г. Оскільки обидві ці задачі еліптичні в $\Omega$, то простори $N$ і $N_{\star}$ скінченновимірні [5] (наслідок 4.1.1). 
3. Простори Хермандера. Еліптичну крайову задачу (1), (2) досліджуємо у придатних парах гільбертових просторів Хермандера $H^{s, \varphi}$, для яких показниками регулярності (або гладкості) служать довільні число $s \in \mathbb{R}$ і функція $\varphi \in \mathcal{M}$. Ці простори утворюють уточнену соболєвську шкалу, введену і досліджену в $[14,15]$. Тут і надалі $\mathcal{M}$ - множина всіх вимірних за Борелем функцій $\varphi:[1, \infty) \rightarrow(0, \infty)$, які обмежені і відокремлені від нуля на кожному компакті та повільно змінюються на нескінченності за Й. Караматою, тобто $\varphi(\lambda t) / \varphi(t) \rightarrow 1$ при $t \rightarrow \infty$ для кожного $\lambda>0$. Повільно змінні функції добре вивчені і мають різноманітні застосування $[38,39]$. Їх характерним прикладом служить функція

$$
\varphi(t):=(\log t)^{r_{1}}(\log \log t)^{r_{2}} \ldots(\underbrace{\log \ldots \log t}_{k \text { разів }})^{r_{k}}, \quad t \gg 1,
$$

де довільно вибрано ціле число $k \geq 1$ і дійсні числа $r_{1}, \ldots, r_{k}$.

Нехай $s \in \mathbb{R}$ i $\varphi \in \mathcal{M}$. Наведемо означення просторів $H^{s, \varphi}$ спочатку для $\mathbb{R}^{n}$, а потім для $\Omega$ і $\Gamma$, та зазначимо деякі їх властивості, потрібні нам. При цьому будемо слідувати монографії [9] (пп. 1.3, 2.1, 3.2).

За означенням, лінійний простір $H^{s, \varphi}\left(\mathbb{R}^{n}\right)$, де ціле $n \geq 1$, складається з усіх розподілів $w \in \mathcal{S}^{\prime}\left(\mathbb{R}^{n}\right)$ таких, що їх перетворення Фур'є $\widehat{w} є$ функцією, яка локально інтегровна на $\mathbb{R}^{n}$ за Лебегом і задовольняє умову

$$
\int_{\mathbb{R}^{n}}\langle\xi\rangle^{2 s} \varphi^{2}(\langle\xi\rangle)|\widehat{w}(\xi)|^{2} d \xi<\infty
$$

Тут, як звичайно, $\mathcal{S}^{\prime}\left(\mathbb{R}^{n}\right)$ - лінійний топологічний простір усіх повільно зростаючих розподілів на $\mathbb{R}^{n}$, а $\langle\xi\rangle:=\left(1+|\xi|^{2}\right)^{1 / 2}$. У просторі $H^{s, \varphi}\left(\mathbb{R}^{n}\right)$ уведено скалярний добуток розподілів $w_{1}$ і $w_{2}$ за формулою

$$
\left(w_{1}, w_{2}\right)_{H^{s, \varphi}\left(\mathbb{R}^{n}\right)}:=\int_{\mathbb{R}^{n}}\langle\xi\rangle^{2 s} \varphi^{2}(\langle\xi\rangle) \widehat{w_{1}}(\xi) \overline{\widehat{w}_{2}(\xi)} d \xi .
$$

Він породжує норму

$$
\|w\|_{H^{s, \varphi\left(\mathbb{R}^{n}\right)}}:=(w, w)_{H^{s, \varphi}\left(\mathbb{R}^{n}\right)}^{1 / 2} .
$$

Простір $H^{s, \varphi}\left(\mathbb{R}^{n}\right)$ - ізотропний гільбертів випадок простору $\mathcal{B}_{p, k}$, введеного і дослідженого Л. Хермандером [6] (п. 2.2). А саме, $H^{s, \varphi}\left(\mathbb{R}^{n}\right)=\mathcal{B}_{2, k}$, якщо $k(\xi)=\langle\xi\rangle^{s} \varphi(\langle\xi\rangle)$ для довільного $\xi \in \mathbb{R}^{n}$.

У важливому окремому випадку, коли $\varphi(\cdot) \equiv 1$, простір $H^{s, \varphi}\left(\mathbb{R}^{n}\right)$ стає гільбертовим простором Соболєва $H^{s}\left(\mathbb{R}^{n}\right)$ порядку s. У загальній ситуації виконуються неперервні та щільні вкладення

$$
H^{s+\varepsilon}\left(\mathbb{R}^{n}\right) \hookrightarrow H^{s, \varphi}\left(\mathbb{R}^{n}\right) \hookrightarrow H^{s-\varepsilon}\left(\mathbb{R}^{n}\right) \quad \text { для кожного } \varepsilon>0 .
$$

3 них випливає, що у класі функціональних просторів $\left\{H^{s, \varphi}\left(\mathbb{R}^{n}\right): s \in \mathbb{R}, \varphi \in \mathcal{M}\right\}$ числовий параметр $s$ задає основну регулярність (або гладкість) розподілів, а функціональний параметр $\varphi$ задає додаткову регулярність, яка уточнює основну. Тому цей клас природно називати уточненою соболєвською шкалою на $\mathbb{R}^{n}$.

Ії̈ аналоги для евклідової області $\Omega$ і замкненого компактного многовиду $Г$ вводяться у стандартний спосіб. Наведемо відповідні означення. 
За означенням, лінійний простір $H^{s, \varphi}(\Omega)$ складається зі звужень в область $\Omega$ усіх розподілів $w \in H^{s, \varphi}\left(\mathbb{R}^{n}\right)$. Норма у ньому означена за формулою

$$
\|u\|_{H^{s, \varphi}(\Omega)}:=\inf \left\{\|w\|_{H^{s, \varphi}\left(\mathbb{R}^{n}\right)}: w \in H^{s, \varphi}\left(\mathbb{R}^{n}\right), w=u \text { в } \Omega\right\},
$$

де $u \in H^{s, \varphi}(\Omega)$. Цей простір гільбертів і сепарабельний відносно вказаної норми та неперервно вкладений у топологічний простір $\mathcal{D}^{\prime}(\Omega)$ усіх розподілів в $\Omega$. Множина $C^{\infty}(\bar{\Omega})$ щільна у просторі $H^{s, \varphi}(\Omega)$. Він є окремим випадком гільбертових просторів, уведених і досліджених Л. Р. Волевичим і Б. П. Панеяхом [40, § 2].

Коротко кажучи, простір $H^{s, \varphi}(\Gamma)$ складається з усіх розподілів на $Г$, які в локальних координатах дають елементи простору $H^{s, \varphi}\left(\mathbb{R}^{n-1}\right)$. Дамо докладне означення. Нехай довільним чином вибрано скінченний атлас із $C^{\infty}$-структури на многовиді $\Gamma$, утворений локальними картами $\pi_{j}: \mathbb{R}^{n-1} \leftrightarrow \Gamma_{j}$, де $j=1, \ldots, p$. Тут відкриті множини $\Gamma_{1}, \ldots, \Gamma_{p}$ складають скінченне покриття многовиду $Г$. Нехай, окрім того, вибрано функції $\chi_{j} \in C^{\infty}(\Gamma)$, де $j=1, \ldots, p$, які утворюють розбиття одиниці на $\Gamma$, що задовольняє умову $\operatorname{supp} \chi_{j} \subset \Gamma_{j}$.

Тоді, за означенням, лінійний простір $H^{s, \varphi}(\Gamma)$ складається з усіх розподілів $h \in \mathcal{D}^{\prime}(\Gamma)$ таких, що $\left(\chi_{j} h\right) \circ \pi_{j} \in H^{s, \varphi}\left(\mathbb{R}^{n-1}\right)$ для кожного номера $j \in\{1, \ldots, p\}$. Тут, звісно, $\mathcal{D}^{\prime}(\Gamma)-$ лінійний топологічний простір усіх розподілів на $\Gamma$, a $\left(\chi_{j} h\right) \circ \pi_{j}$ є зображенням розподілу $h$ у локальній карті $\pi_{j}$. У просторі $H^{s, \varphi}(\Gamma)$ уведено норму за формулою

$$
\|h\|_{H^{s, \varphi}(\Gamma)}:=\left(\sum_{j=1}^{p}\left\|\left(\chi_{j} h\right) \circ \pi_{j}\right\|_{H^{s, \varphi}\left(\mathbb{R}^{n-1}\right)}^{2}\right)^{1 / 2} .
$$

Цей простір гільбертів і сепарабельний відносно цієї норми та неперервно вкладений у $\mathcal{D}^{\prime}(\Gamma)$. Важливо, що простір $H^{s, \varphi}(\Gamma)$ з точністю до еквівалентності норм не залежить від зазначеного вибору атласу і розбиття одиниці (див. [9] (теорема 2.3)). Множина $C^{\infty}(\Gamma)$ щільна у $H^{s, \varphi}(\Gamma)$.

Введені гільбертові функціональні простори утворюють уточнені соболєвські шкали

$$
\left\{H^{s, \varphi}(\Omega): s \in \mathbb{R}, \varphi \in \mathcal{M}\right\} \quad \text { i } \quad\left\{H^{s, \varphi}(\Gamma): s \in \mathbb{R}, \varphi \in \mathcal{M}\right\}
$$

на $\Omega$ і Г відповідно. Ці шкали є двобічними за числовим параметром $s$. Вони містять двобічні гільбертові соболєвські шкали: якщо $\varphi(\cdot) \equiv 1$, то $H^{s, \varphi}(\Omega)=: H^{s}(\Omega)$ і $H^{s, \varphi}(\Gamma)=$ : $H^{s}(\Gamma)$ є простори Соболєва порядку $s \in \mathbb{R}$. Для шкал $(7)$ виконуються компактні і щільні вкладення (6), якщо у формулі (6) замінити $\mathbb{R}^{n}$ на $\Omega$ або Г відповідно.

Обговоримо зв'язок між шкалами (7). Нехай $s>1 / 2$ і $\varphi \in \mathcal{M}$; тоді відображення сліду $u \mapsto u\left\lceil\Gamma\right.$, де $u \in C^{\infty}(\Gamma)$, продовжується єдиним чином (за неперервністю) до обмеженого лінійного оператора $R_{\Gamma}: H^{s, \varphi}(\Omega) \rightarrow H^{s-1 / 2, \varphi}(\Gamma)$. Отже, для кожного розподілу $u \in H^{s, \varphi}(\Omega)$, його слід $R_{\Gamma} u$ на Г означений коректно. Більше того,

$$
H^{s-1 / 2, \varphi}(\Gamma)=\left\{R_{\Gamma} u: u \in H^{s, \varphi}(\Omega)\right\}
$$

та виконується еквівалентність норм

$$
\|h\|_{H^{s-1 / 2, \varphi}(\Gamma)} \asymp \inf \left\{\|u\|_{H^{s, \varphi}(\Omega)}: u \in H^{s, \varphi}(\Omega), \quad h=R_{\Gamma} u\right\}
$$

на класі всіх функцій $h \in H^{s-1 / 2, \varphi}(\Gamma)$ (див. [9] (теорема 3.5 і наслідок 3.1)). Проте, якщо $s<1 / 2$, то не можна коректно означити слід на $\Gamma$ довільного розподілу $u \in H^{s, \varphi}(\Omega)$. А саме, відображення $u \mapsto u \uparrow \Gamma$, де $u \in C^{\infty}(\bar{\Omega})$, не можна продовжити до неперервного 
лінійного оператора $R_{\Gamma}: H^{s, \varphi}(\Omega) \rightarrow \mathcal{D}^{\prime}(\Gamma)$ (див. [9] (зауваження 3.5)). Це застереження зберігає силу і для $s=1 / 2$ у соболєвському випадку $\varphi(\cdot) \equiv 1$.

4. Основні результати роботи стосуються характеру розв'язності еліптичної крайової задачі (1), (2) і регулярності їі узагальнених розв'язків у просторах Хермандера, які утворюють двобічні шкали (7). 3 огляду на зазначений вище зв'язок між цими шкалами розглянемо окремо випадки $s>m+1 / 2$ і $s \leq m+1 / 2$. У першому з них нами доведено такий результат [27] (теорема 1).

Твердження 1. Нехай $s>m+1 / 2 i \varphi \in \mathcal{M}$. Тоді відображення (3) продовжуєтъся единим чином (за неперервністю) до обмеженого лінійного оператора

$$
(A, B): H^{s, \varphi}(\Omega) \rightarrow H^{s-2 q, \varphi}(\Omega) \oplus \bigoplus_{j=1}^{q} H^{s-m_{j}-1 / 2, \varphi}(\Gamma)=: \mathcal{H}_{s-2 q, s, \varphi}(\Omega, \Gamma)
$$

Цей оператор нетерів. Його ядро дорівнюе $N$, а область значень складаєтъся з усіх векторів $\left(f, g_{1}, \ldots, g_{q}\right) \in \mathcal{H}_{s-2 q, s, \varphi}(\Omega, \Gamma)$ таких, що

$$
\begin{aligned}
& (f, v)_{\Omega}+\sum_{j=1}^{r-2 q}\left(D_{\nu}^{j-1} f, w_{j}\right)_{\Gamma}+\sum_{j=1}^{q}\left(g_{j}, h_{j}\right)_{\Gamma}=0 \\
& \text { для всіх } \quad\left(v, w_{1}, \ldots, w_{r-2 q}, h_{1}, \ldots, h_{q}\right) \in N_{\star} .
\end{aligned}
$$

Індекс оператора (8) дорівнюе $\operatorname{dim} N-\operatorname{dim} N_{\star}$ та не залежить від s $i \varphi$.

У зв'язку з цим твердженням нагадаємо, що лінійний обмежений оператор $T: E_{1} \rightarrow$ $E_{2}$, де $E_{1}$ і $E_{2}$ - банахові простори, називають нетеровим, якщо його ядро $\operatorname{ker} T$ i коядро $E_{2} / T\left(E_{1}\right)$ скінченновимірні. Якщо цей оператор нетерів, то його область значень замкнена в $E_{2}$ і він має скінченний індекс

$$
\text { ind } T:=\operatorname{dim} \operatorname{ker} T-\operatorname{dim}\left(E_{2} / T\left(E_{1}\right)\right) \text {. }
$$

Зі сказаного наприкінці п. 3 випливає, що умову $s>m+1 / 2$ у твердженні 1 не можна відкинути чи послабити. Зокрема, якщо $s \leq m+1 / 2$ і $\varphi(\cdot) \equiv 1$, то відображення $u \mapsto B_{j} u$, де $u \in C^{\infty}(\bar{\Omega})$, не можна продовжити до неперервного лінійного оператора $B_{j}: H^{s}(\Omega) \rightarrow \mathcal{D}^{\prime}(\Gamma)$ у випадку, коли $m_{j}=m$.

Для того, щоб отримати версію твердження 1 для довільного $s \leq m+1 / 2$, обмежимося розглядом розв'язків $u \in H^{s, \varphi}(\Omega)$ еліптичного рівняння $A u=f$, права частина якого належить до простору

$$
H^{m+1 / 2-2 q+}(\Omega):=\bigcup_{\lambda>m+1 / 2-2 q} H^{\lambda}(\Omega)=\bigcup_{\substack{\lambda>m+1 / 2-2 q, \eta \in \mathcal{M}}} H^{\lambda, \eta}(\Omega)
$$

(тут друга рівність виконується з огляду на вкладення (6)).

Нехай $s \leq m+1 / 2, \varphi \in \mathcal{M}$ і $\lambda>m+1 / 2-2 q$. Розглянемо лінійний простір

$$
H_{A, \lambda}^{s, \varphi}(\Omega):=\left\{u \in H^{s, \varphi}(\Omega): A u \in H^{\lambda}(\Omega)\right\},
$$

наділений нормою графіка

$$
\|u\|_{H_{A, \lambda}^{s, \varphi}(\Omega)}:=\left(\|u\|_{H^{s, \varphi}(\Omega)}^{2}+\|A u\|_{H^{\lambda}(\Omega)}^{2}\right)^{1 / 2} .
$$

Тут $A u$ розуміємо в сенсі теорії розподілів в області $\Omega$. У соболєвському випадку $\varphi(\cdot) \equiv 1$ будемо пропускати індекс $\varphi$ у позначеннях цього та інших просторів, введених на основі просторів Хермандера $H^{s, \varphi}$. 
Цей простір гільбертів відносно норми (10). Справді, ця норма породжена скалярним добутком, оскільки такими є норми в правій частині рівності (10). Окрім того, простір $H_{A, \lambda}^{s, \varphi}(\Omega)$ повний відносно цієї норми. Справді, якщо послідовність $\left(u_{k}\right)$ фундаментальна в цьому просторі, то існують границі $u:=\lim u_{k}$ в $H^{s, \varphi}(\Omega)$ і $f:=\lim A u_{k}$ в $H^{\lambda}(\Omega)$, оскільки останні два простори повні. Диференціальний оператор $A$ неперервний у $\mathcal{D}^{\prime}(\Omega)$; тому $A u=\lim A u_{k}=f$ в $\mathcal{D}^{\prime}(\Omega)$. Тут, нагадаємо, $u \in H^{s, \varphi}(\Omega)$ і $f \in H^{\lambda}(\Omega)$. Тому $u \in H_{A, \lambda}^{s, \varphi}(\Omega)$ i $\lim u_{k}=u$ у просторі $H_{A, \lambda}^{s, \varphi}(\Omega)$. Отже, цей простір повний.

Теорема 1. Нехай $s \leq m+1 / 2, \varphi \in \mathcal{M} i \lambda>m+1 / 2-2 q$. Тоді множина $C^{\infty}(\bar{\Omega})$ щільна в просторі $H_{A, \lambda}^{s, \varphi}(\Omega)$, а відображення (3) продовжується єдиним чином (за неперервністю) до обмеженого лінійного оператора

$$
(A, B): H_{A, \lambda}^{s, \varphi}(\Omega) \rightarrow H^{\lambda}(\Omega) \oplus \bigoplus_{j=1}^{q} H^{s-m_{j}-1 / 2, \varphi}(\Gamma)=: \mathcal{H}_{\lambda, s, \varphi}(\Omega, \Gamma)
$$

Цей оператор нетерів. Його ядро дорівнюе $N$, а область значень складаеться з усіх векторів $\left(f, g_{1}, \ldots, g_{q}\right) \in \mathcal{H}_{\lambda, s, \varphi}(\Omega, \Gamma)$, які задовольняють умову (9). Індекс оператора (11) дорівнюе $\operatorname{dim} N-\operatorname{dim} N_{\star}$ та не залежить від $s, \varphi i \lambda$.

Перейдемо до питання про регулярність узагальнених розв'язків крайової задачі (1), (2) у двобічній уточненій соболєвській шкалі. Спочатку дамо означення цих розв'язків. Позначимо через $\mathcal{S}^{\prime}(\Omega)$ лінійний простір звужень в область $\Omega$ усіх розподілів $w \in \mathcal{S}^{\prime}\left(\mathbb{R}^{n}\right)$. Покладемо

$$
\mathcal{S}_{A, m+1 / 2-2 q+}^{\prime}(\Omega):=\left\{u \in \mathcal{S}^{\prime}(\Omega): A u \in H^{m+1 / 2-2 q+}(\Omega)\right\} .
$$

Нехай розподіл $u \in \mathcal{S}_{A, m+1 / 2-2 q+}^{\prime}(\Omega)$; тоді $u \in H_{A, \lambda}^{s}(\Omega)$ для деяких чисел $s \leq m+1 / 2$ і $\lambda>m+1 / 2-2 q$. Розподіл $u$ називаємо (сильним) узагальненим розв'язком крайової задачі (1), (2) з правою частиною

$$
\left(f, g_{1}, \ldots, g_{q}\right) \in \mathcal{S}^{\prime}(\Omega) \times\left(\mathcal{D}^{\prime}(\Gamma)\right)^{q}
$$

якщо $(A, B) u=\left(f, g_{1}, \ldots, g_{q}\right)$, де $(A, B)$ - оператор (11). Звісно, це означення не залежить від вибору чисел $s$ і $\lambda$.

Нехай $V-$ відкрита множина в $\mathbb{R}^{n}$, яка задовольняє умову $\Omega_{0}:=\Omega \cap V \neq \varnothing$. Покладемо $\Gamma_{0}:=\Gamma \cap V$ (можливий випадок, коли $\left.\Gamma_{0}=\varnothing\right)$. Уведемо для множин $\Omega_{0}$ і $\Gamma_{0}$ локальні аналоги просторів $H^{\sigma, \varphi}(\Omega)$ і $H^{\sigma, \varphi}(\Gamma)$, де $\sigma \in \mathbb{R}$ i $\varphi \in \mathcal{M}$. За означенням, лінійний простір $H_{\text {loc }}^{\sigma, \varphi}\left(\Omega_{0}, \Gamma_{0}\right)$ складається з усіх розподілів $u \in \mathcal{S}^{\prime}(\Omega)$ таких, що $\chi u \in H^{\sigma, \varphi}(\Omega)$ для довільної функції $\chi \in C^{\infty}(\bar{\Omega})$, носій якої задовольняє умову supp $\chi \subset \Omega_{0} \cup \Gamma_{0}$. Аналогічно, лінійний простір $H_{\mathrm{loc}}^{\sigma, \varphi}\left(\Gamma_{0}\right)$ складається, за означенням, з усіх розподілів $h \in \mathcal{D}^{\prime}(\Gamma)$ таких, що $\chi h \in H^{\sigma, \varphi}(\Gamma)$ для довільної функції $\chi \in C^{\infty}(\Gamma)$, носій якої задовольняє умову $\operatorname{supp} \chi \subset \Gamma_{0}$.

Теорема 2. Нехай $s \in \mathbb{R} i \varphi \in \mathcal{M}$. Припустимо, що розподіл $u \in \mathcal{S}_{A, m+1 / 2-2 q+}^{\prime}(\Omega) \epsilon$ узагальненим розв'язком еліптичної крайової задачі (1), (2), праві частини якої задовольняють умови $f \in H_{\mathrm{loc}}^{s-2 q, \varphi}\left(\Omega_{0}, \Gamma_{0}\right) i g_{j} \in H_{\mathrm{loc}}^{s-m_{j}-1 / 2, \varphi}\left(\Gamma_{0}\right)$ для кожного $j \in\{1, \ldots, q\}$. Tодi $u \in H_{\mathrm{loc}}^{s, \varphi}\left(\Omega_{0}, \Gamma_{0}\right)$.

Як бачимо, уточнена регулярність $\varphi \in \mathcal{M}$ правих частин досліджуваної задачі успадковується її узагальненим розв'язком. Відмітимо важливі окремі випадки теореми 2. Якщо $\Omega_{0}=\Omega$ і $\Gamma_{0}=\Gamma$, то простори $H_{\mathrm{loc}}^{\sigma, \varphi}\left(\Omega_{0}, \Gamma_{0}\right)$ і $H_{\mathrm{loc}}^{\sigma, \varphi}\left(\Gamma_{0}\right)$ збігаються з просторами $H^{\sigma, \varphi}(\Omega)$ і $H^{\sigma, \varphi}(\Gamma)$ відповідно. У цьому випадку теорема 2 стверджує, що регулярність узагальненого розв'язку $и$ підвищується глобально, тобто в усій області $\Omega$ аж до їі межі Г. Якщо $\Gamma_{0}=\varnothing$, то регулярність розв'язку $u$ підвищується в околах усіх точок 
$x \in \Omega_{0}$ за умови, що ці околи не перетинають межу підобласті $\Omega_{0}$. У випадку $\Gamma_{0}=\varnothing$ простір $H_{\mathrm{loc}}^{\sigma, \varphi}\left(\Gamma_{0}\right)$ збігається з $\mathcal{D}^{\prime}(\Gamma)$ і тому умова на $g_{j}$ у теоремі 2 стає тривіальною. У цьому випадку висновок теореми 2 випливає з [9] (теорема 4.19). Отже,

$$
\mathcal{S}_{A, m+1 / 2-2 q+}^{\prime}(\Omega) \subset \bigcup_{\sigma>m+1 / 2} H_{\mathrm{loc}}^{\sigma}(\Omega, \varnothing) .
$$

Теореми 1 і 2 є новими навіть у соболєвському випадку, коли $\varphi(\cdot) \equiv 1$.

5. Доведення основних результатів. Обгрунтуємо основні результати роботи теореми 1 і 2.

Доведення теореми 1. Спочатку обгрунтуємо її у соболєвському випадку, коли $\varphi(\cdot) \equiv 1$ і ціле $s<2 q$. У цьому випадку щільність множини $C^{\infty}(\bar{\Omega})$ у просторі $H_{A, \lambda}^{s}(\Omega)$, випливає з теорем 4.25(i) та 4.26 з монографії [9] (див. також [33], теореми 1(i) та 2). Справді, за другою з них простір $H^{\lambda}(\Omega)$ задовольняє умову $\mathrm{I}_{s-2 q}$, сформульовану в п. 4.4.2 цієї монографії. Тому за першою з цих теорем множина

$$
\left\{u \in C^{\infty}(\bar{\Omega}): A u \in H^{\lambda}(\Omega)\right\}=C^{\infty}(\bar{\Omega})
$$

щільна в просторі $H_{A, \lambda}^{s}(\Omega)$.

Для доведення решти теореми 1 у розглянутому випадку скористаємося таким результатом про розв'язність досліджуваної задачі (1), (2) у просторах СоболєваРойтберга: відображення (3) продовжується єдиним чином (за неперервністю) до нетеревого обмеженого лінійного оператора

$$
(A, B): H^{s,(r)}(\Omega) \rightarrow H^{s-2 q,(r-2 q)}(\Omega) \oplus \bigoplus_{j=1}^{q} H^{s-m_{j}-1 / 2}(\Gamma)=: \mathcal{H}_{s-2 q, s}^{(r-2 q)}(\Omega, \Gamma) .
$$

Тут через $H^{\sigma,(k)}(\Omega)$, де $\sigma \in \mathbb{R}$ i $1 \leq k \in \mathbb{Z}$, позначено гільбертів функціональний простір, введений Я. А. Ройтбергом [41] на основі соболєвських просторів (див. також його монографію [4] (п. 2.1)). За означенням, простір Соболєва-Ройтберга $H^{\sigma,(k)}(\Omega)$, де $\sigma \notin\{1 / 2, \ldots, k-1 / 2\}, \epsilon$ поповненням лінійного многовиду $C^{\infty}(\bar{\Omega})$ за гільбертовою нормою

$$
\|u\|_{H^{\sigma,(k)}(\Omega)}:=\left(\|u\|_{H^{\sigma,(0)}(\Omega)}^{2}+\sum_{j=1}^{k} \|\left(D_{\nu}^{j-1} u\right)\left\lceil\Gamma \|_{H^{\sigma-j+1 / 2}(\Gamma)}^{2}\right)^{1 / 2} .\right.
$$

Тут $H^{\sigma,(0)}(\Omega):=H^{\sigma}(\Omega)$, якщо $\sigma \geq 0$, та $H^{\sigma,(0)}(\Omega)-$ поповнення $C^{\infty}(\bar{\Omega})$ за гільбертовою нормою

$$
\|u\|_{H^{\sigma,(0)}(\Omega)}:=\sup \left\{\left|(u, v)_{\Omega}\right|: v \in H^{-\sigma}(\Omega),\|v\|_{H^{-\sigma}(\Omega)}=1\right\}
$$

якщо $\sigma<0$. У випадку, коли $\sigma \in\{1 / 2, \ldots, k-1 / 2\}$, простір $H^{\sigma,(k)}(\Omega)$ означається шляхом інтерполяції з параметром $1 / 2$ пари гільбертових просторів $H^{\sigma \mp \varepsilon,(k)}(\Omega)$, де $0<$ $\varepsilon<1$.

Простір $H^{\sigma,(k)}(\Omega)$, де $\sigma \notin\{1 / 2, \ldots, k-1 / 2\}$, допускає такий опис [4] (лема 2.2.1): лінійне відображення

$$
T_{k}: u \mapsto\left(u, u \uparrow \Gamma, \ldots,\left(D_{\nu}^{k-1} u\right) \uparrow \Gamma\right), \quad \text { де } \quad u \in C^{\infty}(\bar{\Omega}),
$$

продовжується єдиним чином (за неперервністю) до ізометричного оператора

$$
T_{k}: H^{\sigma,(k)}(\Omega) \rightarrow H^{\sigma,(0)}(\Omega) \oplus \bigoplus_{j=1}^{k} H^{\sigma-j+1 / 2}(\Gamma)=: \Pi_{\sigma,(k)}(\Omega, \Gamma)
$$


область значень якого складається з усіх векторів $\left(u_{0}, u_{1}, \ldots, u_{k}\right) \in \Pi_{\sigma,(k)}(\Omega, \Gamma)$ таких, що $u_{j}=R_{\Gamma} D_{\nu}^{j-1} u_{0}$ для кожного $j \in\{1, \ldots, k\}$, що задовольняє умову $\sigma>j-1 / 2$.

Зауважимо [4] (п. 2.1), що при $\sigma>k-1 / 2$ простори $H^{\sigma,(k)}(\Omega)$ і $H^{\sigma}(\Omega)$ рівні як поповнення лінійного многовиду $C^{\infty}(\bar{\Omega})$ за еквівалентними нормами. Окрім того, виконується неперервне вкладення $H^{\sigma,(k)}(\Omega) \hookrightarrow H^{\theta,(k)}(\Omega)$, якщо $\theta<\sigma$.

Обмеженість і нетеровість оператора (12) доведена Ю. В. Костарчуком і Я. А. Ройтбергом в [32] (теорема 5) для довільного дійсного $s$. Там же показано, що $N-$ ядро цього оператора. Згідно з [4] (теорема 4.1.3) область значень оператора (12) складається з усіх векторів $\left(f, g_{1}, \ldots, g_{q}\right) \in \mathcal{H}_{s-2 q, s}^{(r-2 q)}(\Omega, \Gamma)$ таких, що

$$
\begin{gathered}
\left(f_{0}, v\right)_{\Omega}+\sum_{j=1}^{r-2 q}\left(f_{j}, w_{j}\right)_{\Gamma}+\sum_{j=1}^{q}\left(g_{j}, h_{j}\right)_{\Gamma}=0 \\
\text { для всіх }\left(v, w_{1}, \ldots, w_{r-2 q}, h_{1}, \ldots, h_{q}\right) \in \mathcal{N}_{\star},
\end{gathered}
$$

а індекс дорівнюе $\operatorname{dim} N-\operatorname{dim} \mathcal{N}_{\star}$. Тут $\left(f_{0}, f_{1}, \ldots, f_{r-2 q}\right):=T_{r-2 q} f$, а $\mathcal{N}_{\star} \in$ деяким скінченновимірним простором, який лежить в $C^{\infty}(\bar{\Omega}) \times\left(C^{\infty}(\Gamma)\right)^{r-q}$ та не залежить від $s$. Згідно з [5] (теорема 4.1.4) можна покласти $\mathcal{N}_{\star}:=N_{\star}$ в описі (13) області значень оператора (12) для цілих $s$. Покажемо, що те саме можна зробити і у формулі індексу цього оператора. Це достатньо показати для $s=0$ з огляду на незалежність індексу від $s$. Оскільки $N_{\star} \subset C^{\infty}(\bar{\Omega}) \times\left(C^{\infty}(\Gamma)\right)^{r-q}$, то $N_{\star}$ можна розглядати як скінченновимірний підпростір простору

$$
H^{2 q}(\Omega) \oplus \bigoplus_{j=1}^{r-2 q} H^{2 q+j-1 / 2}(\Gamma) \oplus \bigoplus_{j=1}^{q} H^{m_{j}+1 / 2}(\Gamma)
$$

Останній є взаємно спряженим до простору

$$
\Pi_{-2 q,(r-2 q)}(\Omega, \Gamma) \oplus \bigoplus_{j=1}^{q} H^{-m_{j}-1 / 2}(\Gamma)
$$

відносно розширення за неперервністю скалярного добутку в $L_{2}(\Omega) \oplus\left(L_{2}(\Gamma)\right)^{r-q}$. За такого розгляду простір, спряжений до $N_{\star}$, збігається з факторпростором простору (14) за підпростором усіх векторів

$$
\left(f_{0}, f_{1}, \ldots, f_{r-2 q}, g_{1}, \ldots, g_{q}\right) \in \Pi_{-2 q,(r-2 q)}(\Omega, \Gamma) \oplus \bigoplus_{j=1}^{q} H^{-m_{j}-1 / 2}(\Gamma),
$$

які задовольняють умову (13), де беремо $\mathcal{N}_{\star}:=N_{\star}$. Звідси, оскільки оператор $T_{r-2 q}$ здійснює ізоморфізм простору $H^{-2 q,(r-2 q)}(\Omega)$ на простір $\Pi_{-2 q,(r-2 q)}(\Omega, \Gamma)$, випливає, що ковимірність області значень оператора (12), де $s=0$, дорівнюе вимірності цього факторпростору, тобто становить $\operatorname{dim} N_{\star}$. Отже, індекс цього оператора дорівнює $\operatorname{dim} N-\operatorname{dim} N_{\star}$.

Покладемо

$$
H_{A, \lambda}^{s,(r)}(\Omega):=\left\{u \in H^{s,(r)}(\Omega): A u \in H^{\lambda}(\Omega)\right\} .
$$

Тут для кожного $u \in H^{s,(r)}(\Omega)$ елемент $A u \in H^{s-2 q,(r-2 q)}(\Omega)$ означено за допомогою оператора (12). Для цього елемента умова $A u \in H^{\lambda}(\Omega)$ має сенс, оскільки, як зазначалося вище,

$$
H^{\lambda}(\Omega)=H^{\lambda,(r-2 q)}(\Omega) \hookrightarrow H^{s-2 q,(r-2 q)}(\Omega),
$$


причому вкладення неперервне. Наділимо лінійний простір $H_{A, \lambda}^{s,(r)}(\Omega)$ нормою графіка

$$
\|u\|_{H_{A, \lambda}^{s,(r)}(\Omega)}:=\left(\|u\|_{H^{s,(r)}(\Omega)}^{2}+\|A u\|_{H^{\lambda}(\Omega)}^{2}\right)^{1 / 2} .
$$

Цей простір гільбертів відносно норми (16). Справді, ця норма, звісно, породжена деяким скалярним добутком. Окрім того, простір $H_{A, \lambda}^{s,(r)}(\Omega)$ повний відносно неї. Дійсно, якщо послідовність $\left(u_{k}\right)$ фундаментальна в цьому просторі, то існують границі $u:=\lim u_{k}$ в $H^{s,(r)}(\Omega)$ і $f:=\lim A u_{k}$ в $H^{\lambda}(\Omega)$, оскільки останні два простори повні. 3 першої границі випливає, що $A u=\lim A u_{k}$ в $H^{s-2 q,(r-2 q)}(\Omega)$. Звідси на підставі формули (15) і другої границі маємо рівність $A u=f$. Тому $u \in H_{A, \lambda}^{s,(r)}(\Omega)$ i $\lim u_{k}=u$ у просторі $H_{A, \lambda}^{s,(r)}(\Omega)$. Отже, цей простір повний.

Звуження відображення (12) на простір $H_{A, \lambda}^{s,(r)}(\Omega)$ є лінійним оператором

$$
(A, B): H_{A, \lambda}^{s,(r)}(\Omega) \rightarrow H^{\lambda}(\Omega) \oplus \bigoplus_{j=1}^{q} H^{s-m_{j}-1 / 2}(\Gamma)=: \mathcal{H}_{\lambda, s}(\Omega, \Gamma)
$$

Із вказаних вище властивостей оператора (12) безпосередньо випливає, що оператор (17) обмежений, його ядро дорівнює $N$, а область значень

$$
(A, B)\left(H_{A, \lambda}^{s,(r)}(\Omega)\right)=\mathcal{H}_{\lambda, s}(\Omega, \Gamma) \cap(A, B)\left(H^{s,(r)}(\Omega)\right) .
$$

3 цієї рівності та замкненості $(A, B)\left(H^{s,(r)}(\Omega)\right)$ у просторі

$$
\mathcal{H}_{s-2 q, s}^{(r-2 q)}(\Omega, \Gamma) \hookleftarrow \mathcal{H}_{\lambda, s}(\Omega, \Gamma)
$$

випливає, що область значень оператора (17) замкнена у просторі $\mathcal{H}_{\lambda, s}(\Omega, \Gamma)$ і складається з усіх векторів $\left(f, g_{1}, \ldots, g_{q}\right) \in \mathcal{H}_{\lambda, s}(\Omega, \Gamma)$, які задовольняють умову (13), де $\left(f_{0}, f_{1}, \ldots, f_{r-2 q}\right):=T_{r-2 q} f$ і $\mathcal{N}_{\star}:=N_{\star}$. Оскільки $f \in H^{\lambda}(\Omega)$ і $\lambda>m+1 / 2-2 q$, то з означення оператора $T_{r-2 q}$ випливає, що $f_{0}=f$ i $f_{j}=R_{\Gamma} D_{\nu}^{j-1} f$ для кожного $j \in\{1, \ldots, r-2 q\}$. Тому умова (13) набирає вигляду (9).

Для обгрунтування нетеровості оператора (17) залишається показати, що його область значень має скінченну ковимірність. Нагадаємо, що ковимірність області значень оператора (12) дорівнює $\operatorname{dim} N_{\star}<\infty$. Окрім того, множина $C^{\infty}(\bar{\Omega}) \times\left(C^{\infty}(\Gamma)\right)^{q}$ щільна в просторі $\mathcal{H}_{s-2 q, s}^{(r-2 q)}(\Omega, \Gamma)$. Тому, за лемою Гохберга-Крейна [42] (лема 2.1), існує скінченновимірний простір $N_{1} \subset C^{\infty}(\bar{\Omega}) \times\left(C^{\infty}(\Gamma)\right)^{q}$ такий, що

$$
\mathcal{H}_{s-2 q, s}^{(r-2 q)}(\Omega, \Gamma)=(A, B)\left(H^{s,(r)}(\Omega)\right) \dot{+} N_{1}
$$

(як звичайно, знак $\dot{+}$ служить для позначення прямої суми підпросторів). При цьому $\operatorname{dim} N_{1}=\operatorname{dim} N_{\star}$. Звуження цієї суми на простір $\mathcal{H}_{\lambda, s}(\Omega, \Gamma)$ дає на підставі формул (18) і $N_{1} \subset \mathcal{H}_{\lambda, s}(\Omega, \Gamma)$ рівність

$$
\mathcal{H}_{\lambda, s}(\Omega, \Gamma)=(A, B)\left(H_{A, \lambda}^{s,(r)}(\Omega)\right) \dot{+} N_{1}
$$

Отже, ковимірність області значень оператора (17) дорівнює $\operatorname{dim} N_{1}=\operatorname{dim} N_{\star}<\infty$. Таким чином, цей оператор нетерів.

Для того, щоб завершити доведення теореми 1 у розглянутому випадку, достатньо показати, що множина $C^{\infty}(\bar{\Omega})$ щільна у просторі $H_{A, \lambda}^{s,(r)}(\Omega)$ та норми у просторах $H_{A, \lambda}^{s}(\Omega)$ 
і $H_{A, \lambda}^{s,(r)}(\Omega)$ еквівалентні на цій щільній множині. Справді, тоді нетерів оператор (17) стає оператором (11) з формулювання цієї теореми.

Доведемо спочатку, що множина $C^{\infty}(\bar{\Omega})$ щільна в $H_{A, \lambda}^{s,(r)}(\Omega)$. Позначимо через $Q_{A, \lambda}^{s,(r)}(\Omega)$ ортогональне доповнення підпростору $N$ у гільбертовому просторі $H_{A, \lambda}^{s,(r)}(\Omega)$. Звуження нетерового оператора $(17)$ на підпростір $Q_{A, \lambda}^{s,(r)}(\Omega)$ є ізоморфізмом

$$
(A, B): Q_{A, \lambda}^{s,(r)}(\Omega) \leftrightarrow(A, B)\left(H_{A, \lambda}^{s,(r)}(\Omega)\right)
$$

тут, звісно, $(A, B)\left(H_{A, \lambda}^{s,(r)}(\Omega)\right)$ трактується як підпростір простору $\mathcal{H}_{\lambda, s}(\Omega, \Gamma)$. Позначимо через $P$ оператор косого проектування простору $\mathcal{H}_{\lambda, s}(\Omega, \Gamma)$ на підпростір $(A, B)\left(H_{A, \lambda}^{s,(r)}(\Omega)\right)$ паралельно підпростору $N_{1}$ з прямої суми (19).

Подамо довільний елемент $u \in H_{A, \lambda}^{s,(r)}(\Omega)$ у вигляді $u=v+w$, де $v \in Q_{A, \lambda}^{s,(r)}(\Omega)$ i $w \in N$. Оскільки множина $C^{\infty}(\bar{\Omega}) \times\left(C^{\infty}(\Gamma)\right)^{q}$ щільна у просторі $\mathcal{H}_{\lambda, s}(\Omega, \Gamma)$, то для вектора $F:=(A, B) v \in \mathcal{H}_{\lambda, s}(\Omega, \Gamma)$ існуе послідовність $\left(F_{k}\right) \subset C^{\infty}(\bar{\Omega}) \times\left(C^{\infty}(\Gamma)\right)^{q}$ така, що $F_{k} \rightarrow F$ у просторі $\mathcal{H}_{\lambda, s}(\Omega, \Gamma)$. Тоді $P F_{k} \rightarrow P F=F$ у цьому просторі, причому $\left(P F_{k}\right) \subset C^{\infty}(\bar{\Omega}) \times\left(C^{\infty}(\Gamma)\right)^{q}$. Отже,

$$
v_{k}:=(A, B)^{-1} P F_{k} \rightarrow(A, B)^{-1} F=v \quad \text { в } Q_{A, \lambda}^{s,(r)}(\Omega) ;
$$

тут через $(A, B)^{-1}$ позначено оператор, обернений до ізоморфізму (20). Оскільки

$$
(A, B) v_{k}=P F_{k} \in \mathcal{H}_{\sigma-2 q, \sigma}^{(r-2 q)}(\Omega, \Gamma) \quad \text { для кожного } \sigma \in \mathbb{R},
$$

то згідно з [4] (теорема 7.1.1) виконується включення

$$
v_{k} \in \bigcap_{\sigma \in \mathbb{R}} H^{\sigma,(r)}(\Omega)=C^{\infty}(\bar{\Omega})
$$

Отже, $C^{\infty}(\bar{\Omega}) \ni v_{k}+w \rightarrow v+w=u$ у просторі $H_{A, \lambda}^{s,(r)}(\Omega)$. 3 огляду на довільність елемента $u \in H_{A, \lambda}^{s,(r)}(\Omega)$ доведено щільність множини $C^{\infty}(\bar{\Omega})$ в $H_{A, \lambda}^{s,(r)}(\Omega)$.

Доведемо тепер, що норми у просторах $H_{A, \lambda}^{s}(\Omega)$ і $H_{A, \lambda}^{s,(r)}(\Omega)$ еквівалентні на $C^{\infty}(\bar{\Omega})$. Зауважимо спочатку, що

$$
\|u\|_{H_{A, \lambda}^{s}(\Omega)} \leq\|u\|_{H_{A, \lambda}^{s,(r)}(\Omega)} \quad \text { для довільного } \quad u \in C^{\infty}(\bar{\Omega}) .
$$

Це випливає з означення норми у просторі $H^{s,(r)}(\Omega)$ : у випадку $s \geq 0-$ безпосередньо, а у випадку $s<0-$ з огляду на те, що

$$
\|u\|_{H^{s}(\Omega)} \leq\|\mathcal{O} u\|_{H^{s}\left(\mathbb{R}^{n}\right)}=\|u\|_{H^{s,(0)}(\Omega)} \quad \text { для довільного } \quad u \in C^{\infty}(\bar{\Omega}) .
$$

Тут $\mathcal{O} u$ позначає продовження нулем на $\mathbb{R}^{n}$ функції $u \in C^{\infty}(\bar{\Omega})$, а рівність правильна, як зазначено в [4, с. 52].

Доведемо обернену оцінку до (21). Для цього розглянемо еліптичну крайову задачу, яка складається з рівняння (1) і крайових умов

$$
D_{\nu}^{j-1} u=g_{j} \quad \text { на } \quad \Gamma, \quad j=1, \ldots, q .
$$

Згідно з [4] (теорема 4.1.1) відображення

$$
u \mapsto(A u, \mathrm{D} u):=\left(A u, u \uparrow \Gamma, \ldots,\left(D_{\nu}^{q-1} u\right) \uparrow \Gamma\right), \quad \text { де } \quad u \in C^{\infty}(\bar{\Omega}),
$$


продовжується єдиним чином (за неперервністю) до нетерового обмеженого лінійного оператора

$$
(A, \mathrm{D}): H^{s,(r)}(\Omega) \rightarrow H^{s-2 q,(r-2 q)}(\Omega) \oplus \bigoplus_{j=1}^{q} H^{s-j+1 / 2}(\Gamma) .
$$

До того ж, ядро $\mathcal{N}$ цього оператора лежить в $C^{\infty}(\bar{\Omega})$. Звуження оператора (24) на простір $H_{A, \lambda}^{s,(r)}(\Omega)$ є нетеровим обмеженим оператором

$$
(A, \mathrm{D}): H_{A, \lambda}^{s,(r)}(\Omega) \rightarrow H^{\lambda}(\Omega) \oplus \bigoplus_{j=1}^{q} H^{s-j+1 / 2}(\Gamma)=: \mathcal{H}_{\lambda, s}^{\mathrm{D}}(\Omega, \Gamma) .
$$

Це доводиться так само як і нетеровість оператора (17).

Крім того, згідно з [9] (теорема 4.27) відображення (23) продовжується єдиним чином (за неперервністю) до нетерового обмеженого лінійного оператора

$$
(A, \mathrm{D}): H_{A, \lambda}^{s}(\Omega) \rightarrow \mathcal{H}_{\lambda, s}^{\mathrm{D}}(\Omega, \Gamma)
$$

причому ядро цього оператора лежить в $C^{\infty}(\bar{\Omega})$ (див. також [33], наслідок 3). Зауважимо, що зазначена теорема доведена в [9] для регулярних еліптичних крайових задач, до яких і належить задача (1), (22). Оператори (25) і (26) мають спільне ядро $\mathcal{N} \subset C^{\infty}(\bar{\Omega})$ і спільну область значень, бо вона є замиканням множини $\left\{(A, \mathrm{D}) u: u \in C^{\infty}(\bar{\Omega})\right\}$ у просторі $\mathcal{H}_{\lambda, s}^{\mathrm{D}}(\Omega, \Gamma)$. Позначимо цю спільну область значень через $\mathcal{R}_{\lambda, s}(\Omega, \Gamma)$.

Нетерові оператори (25) і (26) породжують у канонічний спосіб ізоморфізми

$$
\begin{gathered}
(A, \mathrm{D}): H_{A, \lambda}^{s,(r)}(\Omega) / \mathcal{N} \leftrightarrow \mathcal{R}_{\lambda, s}(\Omega, \Gamma), \\
(A, \mathrm{D}): H_{A, \lambda}^{s}(\Omega) / \mathcal{N} \leftrightarrow \mathcal{R}_{\lambda, s}(\Omega, \Gamma) .
\end{gathered}
$$

Тут, звісно, трактуємо $\mathcal{R}_{\lambda, s}(\Omega, \Gamma)$ як підпростір простору $\mathcal{H}_{\lambda, s}^{\mathrm{D}}(\Omega, \Gamma)$. Для довільної функції $u \in C^{\infty}(\bar{\Omega})$ розглянемо ї̈ клас суміжності $\widetilde{u}:=\{u+w: w \in \mathcal{N}\}$, який належить обом факторпросторам $H_{A, \lambda}^{s,(r)}(\Omega) / \mathcal{N}$ і $H_{A, \lambda}^{s}(\Omega) / \mathcal{N}$. На підставі цих ізоморфізмів маємо еквівалентність норм

$$
\|\widetilde{u}\|_{H_{A, \lambda}^{s,(r)}(\Omega) / \mathcal{N}} \asymp\|(A, \mathrm{D}) \widetilde{u}\|_{\mathcal{R}_{\lambda, s}(\Omega, \Gamma)} \asymp\|\widetilde{u}\|_{H_{A, \lambda}^{s}(\Omega) / \mathcal{N}}
$$

на функціях $u \in C^{\infty}(\bar{\Omega})$. Спираючись на неї, доведену оцінку, обернену до (21).

Для довільного $u \in C^{\infty}(\bar{\Omega})$ існує таке $w \in \mathcal{N}$, що

$$
\|u+w\|_{H_{A, \lambda}^{s,(r)}(\Omega)} \leq 2\|\widetilde{u}\|_{H_{A, \lambda}^{s,(r)}(\Omega) / \mathcal{N}}
$$

Скориставшись еквівалентністю норм на скінченновимірному просторі $\mathcal{N}$ та послідовно формулами (21), (28) і (27), отримаємо такі нерівності:

$$
\begin{gathered}
\|u\|_{H_{A, \lambda}^{s,(r)}(\Omega)} \leq\|u+w\|_{H_{A, \lambda}^{s,(r)}(\Omega)}+\|w\|_{H_{A, \lambda}^{s,(r)}(\Omega)} \leq\|u+w\|_{H_{A, \lambda}^{s,(r)}(\Omega)}+c_{1}\|w\|_{H_{A, \lambda}^{s}(\Omega)} \leq \\
\leq\|u+w\|_{H_{A, \lambda}^{s,(r)}(\Omega)}+c_{1}\|u+w\|_{H_{A, \lambda}^{s}(\Omega)}+c_{1}\|u\|_{H_{A, \lambda}^{s}(\Omega)} \leq \\
\leq\left(1+c_{1}\right)\|u+w\|_{H_{A, \lambda}^{s,(r)}(\Omega)}+c_{1}\|u\|_{H_{A, \lambda}^{s}(\Omega)} \leq 2\left(1+c_{1}\right)\|\widetilde{u}\|_{H_{A, \lambda}^{s,(r)}(\Omega) / \mathcal{N}}+c_{1}\|u\|_{H_{A, \lambda}^{s}(\Omega)} \leq \\
\leq c_{2}\|\widetilde{u}\|_{H_{A, \lambda}^{s}(\Omega) / \mathcal{N}}+c_{1}\|u\|_{H_{A, \lambda}^{s}(\Omega)} \leq\left(c_{2}+c_{1}\right)\|u\|_{H_{A, \lambda}^{s}(\Omega)} ;
\end{gathered}
$$


тут $c_{1}$ i $c_{2}$ - деякі додатні числа, які не залежать від функцій $u$ i $w$. Отже, доведено оцінку, обернену до (21).

Таким чином, теорему 1 доведено у розглянутому випадку, коли $\varphi(\cdot) \equiv 1, s \in \mathbb{Z}$ i $s<2 q$.

У загальній ситуації виведемо цю теорему з розглянутого випадку і твердження 1 за допомогою інтерполяції з функціональним параметром пар гільбертових просторів (означення цієї інтерполяції та потрібні нам її властивості наведено, наприклад, в [9] (п. 1.1) або [43] (п. 2)).

Виберемо ціле число $l \geq 1$, яке задовольняє умови $s>-2 q(l-1)$ і $\lambda<2 q l$. Скористаємося нетеровим оператором (11) у випадку (вже розглянутому), коли $\varphi(\cdot) \equiv 1$ і число $-2 q(l-1)$ узято замість $s$, та нетеровим оператором (8) з твердження 1 у випадку, коли $\varphi(\cdot) \equiv 1$ і число $\lambda+2 q$ узято замість $s$. Отже, отримаємо нетерові обмежені оператори

$$
\begin{gathered}
(A, B): H_{A, \lambda}^{-2 q(l-1)}(\Omega) \rightarrow H^{\lambda}(\Omega) \oplus \bigoplus_{j=1}^{q} H^{-2 q(l-1)-m_{j}-1 / 2}(\Gamma)=\mathcal{H}_{\lambda,-2 q(l-1)}(\Omega, \Gamma), \\
(A, B): H^{\lambda+2 q}(\Omega) \rightarrow H^{\lambda}(\Omega) \oplus \bigoplus_{j=1}^{q} H^{\lambda+2 q-m_{j}-1 / 2}(\Gamma)=\mathcal{H}_{\lambda, \lambda+2 q}(\Omega, \Gamma) .
\end{gathered}
$$

Вони мають спільне ядро $N$ і однаковий індекс, рівний $\operatorname{dim} N-\operatorname{dim} N_{\star}$. Окрім того, перший оператор є розширенням другого.

Покладемо $\varepsilon:=s+2 q(l-1)>0$ i $\delta:=\lambda+2 q-s>0$ та означимо функцію $\psi:(0, \infty) \rightarrow(0, \infty)$ за формулами $\psi(t):=t^{\varepsilon /(\varepsilon+\delta)} \varphi\left(t^{1 /(\varepsilon+\delta)}\right)$, якщо $t \geq 1$, та $\psi(t):=\varphi(1)$, якщо $0<t<1$. Згідно з [9] (теорема 1.14) ця функція є інтерполяційним параметром. Застосувавши інтерполяцію з функціональним параметром $\psi$ до обмежених лінійних операторів (29) і (30), отримаємо обмежений лінійний оператор

$$
(A, B):\left[H_{A, \lambda}^{-2 q(l-1)}(\Omega), H^{\lambda+2 q}(\Omega)\right]_{\psi} \rightarrow\left[\mathcal{H}_{\lambda,-2 q(l-1)}(\Omega, \Gamma), \mathcal{H}_{\lambda, \lambda+2 q}(\Omega, \Gamma)\right]_{\psi} .
$$

Він є звуженням відображення (29) на інтерполяційний простір

$$
\left[H_{A, \lambda}^{-2 q(l-1)}(\Omega), H^{\lambda+2 q}(\Omega)\right]_{\psi}
$$

Тут і далі у доведенні через $\left[X_{0}, X_{1}\right]_{\psi}$ позначено гільбертів простір, який є результатом інтерполяції з функціональним параметром $\psi$ припустимої впорядкованої пари сепарабельних гільбертових просторів $X_{0}$ і $X_{1}$ (див. означення цієї інтерполяції в [9] (п. 1.1.1)). Зауважимо, що цю пару називають припустимою, якщо виконується неперервне і щільне вкладення $X_{1} \hookrightarrow X_{0}$. Тоді також виконуються неперервні і щільні вкладення $X_{1} \hookrightarrow\left[X_{0}, X_{1}\right]_{\psi} \hookrightarrow X_{0}$ (див. [9] (теорема 1.1)). Наведені у формулі (31) пари гільбертових просторів є припустимими. Припустимість першої з них випливає, зокрема, із доведеної вище щільності множини $C^{\infty}(\bar{\Omega})$ у просторі $H_{A, \lambda}^{-2 q(l-1)}(\Omega)$. Крім того, цей простір сепарабельний, що випливає з нетеровості оператора (29) і сепарабельності простору $\mathcal{H}_{\lambda,-2 q(l-1)}(\Omega, \Gamma)$. Припустимість другої пари очевидна.

Множина $C^{\infty}(\bar{\Omega})$ щільна у просторі $(32)$, оскільки у нього неперервно і щільно вкладено простір $H^{\lambda+2 q}(\Omega)$. Тому оператор (31) є продовженням за неперервністю відображення (3). На підставі зазначених вище властивостей нетерових операторів (29) і (30) робимо висновок згідно з теоремою про інтерполяцію нетерових операторів (див. [9] (теорема 1.5)), що оператор (31) нетерів з ядром $N$, індексом $\operatorname{dim} N-\operatorname{dim} N_{\star}$ та областю значень, рівною

$$
\left[\mathcal{H}_{\lambda,-2 q(l-1)}(\Omega, \Gamma), \mathcal{H}_{\lambda, \lambda+2 q}(\Omega, \Gamma)\right]_{\psi} \cap(A, B)\left(H_{A, \lambda}^{-2 q(l-1)}(\Omega)\right) .
$$


3 останньої властивості і вже обгрунтованого опису області значень оператора (29) (з використанням умови (9)) випливає, що область значень оператора (31) складається $з$ усіх векторів

$$
\left(f, g_{1}, \ldots, g_{q}\right) \in\left[\mathcal{H}_{\lambda,-2 q(l-1)}(\Omega, \Gamma), \mathcal{H}_{\lambda, \lambda+2 q}(\Omega, \Gamma)\right]_{\psi},
$$

які задовольняють умову (9).

Для того, щоб завершити доведення теореми 1, залишається показати, що простори, у яких діє нетерів оператор (31), задовольняють рівності

$$
\begin{gathered}
{\left[H_{A, \lambda}^{-2 q(l-1)}(\Omega), H^{\lambda+2 q}(\Omega)\right]_{\psi}=H_{A, \lambda}^{s, \varphi}(\Omega)} \\
{\left[\mathcal{H}_{\lambda,-2 q(l-1)}(\Omega, \Gamma), \mathcal{H}_{\lambda, \lambda+2 q}(\Omega, \Gamma)\right]_{\psi}=\mathcal{H}_{\lambda, s, \varphi}(\Omega, \Gamma)}
\end{gathered}
$$

з точністю до еквівалентності норм.

Рівність (34) випливає з теореми 1.5 (про інтерполяцію прямих сум просторів) і теореми 2.2 (про інтерполяцію з функціональним параметром соболєвських просторів на Г), наведених у [9]. А саме,

$$
\begin{gathered}
{\left[\mathcal{H}_{\lambda,-2 q(l-1)}(\Omega, \Gamma), \mathcal{H}_{\lambda, \lambda+2 q}(\Omega, \Gamma)\right]_{\psi}=} \\
=\left[H^{\lambda}(\Omega), H^{\lambda}(\Omega)\right]_{\psi} \oplus \bigoplus_{j=1}^{q}\left[H^{-2 q(l-1)-m_{j}-1 / 2}(\Gamma), H^{\lambda+2 q-m_{j}-1 / 2}(\Gamma)\right]_{\psi}= \\
=H^{\lambda}(\Omega) \oplus \bigoplus_{j=1}^{q}\left[H^{s-m_{j}-1 / 2-\varepsilon}(\Gamma), H^{s-m_{j}-1 / 2+\delta}(\Gamma)\right]_{\psi}= \\
=H^{\lambda}(\Omega) \oplus \bigoplus_{j=1}^{q} H^{s-m_{j}-1 / 2, \varphi}(\Gamma)=\mathcal{H}_{\lambda, s, \varphi}(\Omega, \Gamma)
\end{gathered}
$$

з точністю до еквівалентності норм.

Для доведення рівності (33) скористаємося одним результатом про інтерполяцію підпросторів, пов'язаних з довільним обмеженим лінійним оператором, який діє в паpi гільбертових просторів. Нехай $H, \Phi$ і $\Psi$ - гільбертові простори, причому виконується неперервне вкладення $\Phi \hookrightarrow \Psi$. Нехай також задано обмежений лінійний оператор $T: H \rightarrow \Psi$. Покладемо $(H)_{T, \Phi}:=\{u \in H: T u \in \Phi\}$. Простір $(H)_{T, \Phi} \in$ гільбертовим відносно норми графіка

$$
\|u\|_{(H)_{T, \Phi}}:=\left(\|u\|_{H}^{2}+\|T u\|_{\Phi}^{2}\right)^{1 / 2} .
$$

Твердження 2. Нехай задано шість сепарабелъних гільбертових просторів $X_{0}$, $Y_{0}, Z_{0}, X_{1}, Y_{1} i Z_{1}$ та три лінійних відображення $T, R$ i $S$, що задовольняють такі сім умов:

(i) napu $X=\left[X_{0}, X_{1}\right]$ i $Y=\left[Y_{0}, Y_{1}\right]$ nрипустимі;

(ii) простори $Z_{0}$ i $Z_{1}$ e підпросторами деякого лінійного простору $E$;

(iii) виконуються неперервні вкладення $Y_{j} \hookrightarrow Z_{j}$ nри $j \in\{0,1\}$;

(iv) відображення $T$ означено на $X_{0} i$ задає обмежені оператори $T: X_{j} \rightarrow Z_{j} n p и$ $j \in\{0,1\}$;

(v) відображсення $R$ означено на $E$ i задае обмежені оператори $R: Z_{j} \rightarrow X_{j} n p u$ $j \in\{0,1\}$;

(vi) відображення $S$ означено на $E$ i задає обмежені оператори $S: Z_{j} \rightarrow Y_{j} n p u$ $j \in\{0,1\}$; 
(vii) для кожного $\omega \in E$ виконуетъся рівність $T R \omega=\omega+S \omega$.

Tодi пара просторів $\left[\left(X_{0}\right)_{T, Y_{0}},\left(X_{1}\right)_{T, Y_{1}}\right]$ припустима $i$ для довільного інтерполяиійного параметра $\psi \in \mathcal{B}$ виконуеться така рівність просторів з точністю до еквівалентності норм:

$$
\left[\left(X_{0}\right)_{T, Y_{0}},\left(X_{1}\right)_{T, Y_{1}}\right]_{\psi}=\left(\left[X_{0}, X_{1}\right]_{\psi}\right)_{T,\left[Y_{0}, Y_{1}\right]_{\psi}} .
$$

Аналог цього твердження був уперше встановлений Ж.-Л. Ліонсом і Е. Мадженесом [30] (теорема 14.3) для комплексної інтерполяції з числовим параметром. Для інтерполяції з функціональним параметром твердження 2 доведено в [16] (п. 4) (див. також [9] (п. 3.3.2)).

У твердженні 2 покладемо $X_{0}:=H^{-2 q(l-1)}(\Omega), X_{1}:=H^{\lambda+2 q}(\Omega), Y_{0}:=Y_{1}:=Z_{1}:=$ $H^{\lambda}(\Omega), Z_{0}:=E:=H^{-2 q l}(\Omega)$ і $T:=A$. Тоді

$$
H_{A, \lambda}^{-2 q(l-1)}(\Omega)=\left(X_{0}\right)_{T, Y_{0}} \quad \text { i } \quad H^{\lambda+2 q}(\Omega)=\left(X_{1}\right)_{T, Y_{1}} .
$$

Зауважимо, що остання рівність виконуеться з точністю до еквівалентності норм, оскільки $A$ є обмеженим оператором у парі просторів $H^{\lambda+2 q}(\Omega)$ і $H^{\lambda}(\Omega)$ (для довільного дійсного $\lambda$ ). Звісно, умови (i) - (iv) твердження 2 виконуються. Побудуємо оператори $R$ і $S$, які задовольняють решту умов (v)-(vii).

Для цього скористаємося тим, що відображення $u \mapsto A^{l} A^{l+} u+u$ задає ізоморфізм

$$
A^{l} A^{l+}+I: H_{\mathrm{D}}^{\sigma}(\Omega) \leftrightarrow H^{\sigma-4 q l}(\Omega) \quad \text { для довільного } \sigma \geq 2 q l
$$

(див., наприклад, лему 3.1 [9], доведення якої проходить і для дійсних $\sigma \geq 2 q l$ ). Тут, як звичайно, $A^{l} \in l$-тою ітерацією оператора $A$, а $A^{l+} \in$ формально спряженим оператором до диференціального оператора $A^{l}$ відносно скалярного добутку в $L_{2}(\Omega)$, та $I-$ тотожний оператор. Окрім того,

$$
H_{\mathrm{D}}^{\sigma}(\Omega):=\left\{u \in H^{\sigma}(\Omega): R_{\Gamma} D_{\nu}^{j-1} u=0 \text { для кожного } j \in\{1, \ldots, 2 q l\}\right\}
$$

є підпростором простору $H^{\sigma}(\Omega)$. Оператор, обернений до $(36)$, є обмеженим лінійним оператором

$$
\left(A^{l} A^{l+}+I\right)^{-1}: H^{\theta}(\Omega) \rightarrow H^{\theta+4 q l}(\Omega) \text { для довільного } \theta \geq-2 q l .
$$

Покладемо

$$
R:=A^{l-1} A^{l+}\left(A^{l} A^{l+}+I\right)^{-1} \quad \text { i } \quad S=-\left(A^{l} A^{l+}+I\right)^{-1} .
$$

Використовуючи (37), одержимо обмежені оператори

$$
\begin{aligned}
& R: Z_{0}=H^{-2 q l}(\Omega) \rightarrow H^{2 q l-2 q l-2 q(l-1)}(\Omega)=X_{0}, \\
& R: Z_{1}=H^{\lambda}(\Omega) \rightarrow H^{\lambda+4 q l-2 q l-2 q(l-1)}(\Omega)=X_{1}, \\
& S: Z_{0}=H^{-2 q l}(\Omega) \rightarrow H^{2 q l}(\Omega) \hookrightarrow H^{\lambda}(\Omega)=Y_{0}, \\
& S: Z_{1}=H^{\lambda}(\Omega) \rightarrow H^{\lambda+4 q l}(\Omega) \hookrightarrow H^{\lambda}(\Omega)=Y_{1} ;
\end{aligned}
$$

тут вкладення неперервні. Окрім того,

$$
A R=A A^{l-1} A^{l+}\left(A^{l} A^{l+}+I\right)^{-1}=\left(A^{l} A^{l+}+I-I\right)\left(A^{l} A^{l+}+I\right)^{-1}=I+S
$$

на просторі $E=H^{-2 q l}(\Omega)$. Таким чином, для введених операторів $R$ і $S$ виконуються умови (v) - (vii) твердження 2. 
Згідно з цим твердженням і на підставі (35), отримаємо рівності

$$
\left[H_{A, \lambda}^{-2 q(l-1)}(\Omega), H^{\lambda+2 q}(\Omega)\right]_{\psi}=\left[\left(X_{0}\right)_{T, Y_{0}},\left(X_{1}\right)_{T, Y_{1}}\right]_{\psi}=\left(\left[X_{0}, X_{1}\right]_{\psi}\right)_{T,\left[Y_{0}, Y_{1}\right]_{\psi}} .
$$

Тут на підставі теореми 3.2 (про інтерполяцію з функціональним параметром соболєвських просторів на $\Omega$ ), наведеної у [9], маємо рівність просторів

$$
\left[X_{0}, X_{1}\right]_{\psi}=\left[H^{-2 q(l-1)}(\Omega), H^{\lambda+2 q}(\Omega)\right]_{\psi}=\left[H^{s-\varepsilon}(\Omega), H^{s+\delta}(\Omega)\right]_{\psi}=H^{s, \varphi}(\Omega) .
$$

Ці рівності виконуються з точністю до еквівалентності норм. Окрім того,

$$
\left[Y_{0}, Y_{1}\right]_{\psi}=\left[H^{\lambda}(\Omega), H^{\lambda}(\Omega)\right]_{\psi}=H^{\lambda}(\Omega)
$$

3 останніх трьох виносних формул негайно випливає потрібна рівність (33).

Теорему 1 доведено.

Доведення теореми 2. Виберемо довільно функцію $\chi \in C^{\infty}(\bar{\Omega})$ таку, що supp $\chi \subset$ $\Omega_{0} \cup \Gamma_{0}$. Виберемо також функцію $\eta \in C^{\infty}(\bar{\Omega})$, яка задовольняє умови supp $\eta \subset \Omega_{0} \cup \Gamma_{0}$ і $\eta=1$ у деякому околі $V$ множини $\operatorname{supp} \chi$ (звісно, цей окіл розглядається у топології на $\bar{\Omega})$. За умовою, $u \in H_{A, \lambda}^{\sigma}(\Omega)$ для деяких цілого числа $\sigma<\min \{s, 2 q\}$ і дійсного числа $\lambda>m+1 / 2-2 q$ та, крім того,

$$
\eta(f, g) \in\left\{\begin{array}{lll}
\mathcal{H}_{\lambda, s, \varphi}(\Omega, \Gamma), & \text { якщо } & s \leq m+1 / 2 \\
\mathcal{H}_{s-2 q, s, \varphi}(\Omega, \Gamma), & \text { якщо } & s>m+1 / 2 .
\end{array}\right.
$$

Тут, звісно, $g:=\left(g_{1}, \ldots, g_{q}\right)$ і $\eta(f, g):=\left(\eta f,(\eta \uparrow \Gamma) g_{1}, \ldots,(\eta \uparrow \Gamma) g_{q}\right)$. У випадку, коли $s>m+1 / 2$, виберемо число $\lambda>m+1 / 2-2 q$ так, щоб додатково виконувалась нерівність $\lambda<s-2 q$.

Взагалі кажучи, $\chi u \notin H_{A, \lambda}^{\sigma}(\Omega)$; тому замість простору $H_{A, \lambda}^{\sigma}(\Omega)$ будемо використовувати більш широкій простір Соболєва-Ройтберга $H^{\sigma,(r)}(\Omega)$, замкнений відносно операції множення на довільну функцію класу $C^{\infty}(\bar{\Omega})$. Як було показано у доведенні теореми 1 , норми у просторах $H_{A, \lambda}^{\sigma}(\Omega)$ і $H_{A, \lambda}^{\sigma,(r)}(\Omega)$ еквівалентні на щільному лінійному многовиді $C^{\infty}(\bar{\Omega})$. Отже, ці простори рівні з точністю до еквівалентності норм і тому виконується неперервне вкладення $H_{A, \lambda}^{\sigma}(\Omega) \hookrightarrow H^{\sigma,(r)}(\Omega)$. Отож, нетерів оператор (12), де беремо число $\sigma$ замість $s, \in$ розширенням нетерового оператора

$$
(A, B): H_{A, \lambda}^{\sigma}(\Omega) \rightarrow \mathcal{H}_{\lambda, \sigma}(\Omega, \Gamma) .
$$

Нагадаємо [4] (наслідок 2.3.1), що оператор множення на функцію класа $C^{\infty}(\bar{\Omega})$ є неперервним на кожному просторі Соболєва - Ройтберга.

Оскільки оператор (39) нетерів, а множина $C^{\infty}(\bar{\Omega}) \times\left(C^{\infty}(\Gamma)\right)^{q}$ щільна в просторі $\mathcal{H}_{\lambda, \sigma}(\Omega, \Gamma)$, то за лемою Гохберга - Крейна [42] (лема 2.1) існує скінченновимірний простір $N_{0} \subset C^{\infty}(\bar{\Omega}) \times\left(C^{\infty}(\Gamma)\right)^{q}$ такий, що

$$
\mathcal{H}_{\lambda, \sigma}(\Omega, \Gamma)=(A, B)\left(H_{A, \lambda}^{\sigma}(\Omega)\right) \dot{+} N_{0}
$$

Позначимо через $P_{0}$ проектор простору $\mathcal{H}_{\lambda, \sigma}(\Omega, \Gamma)$ на перший доданок у цій прямій сумі паралельно другому доданку.

На підставі умови (38), теореми 1 і твердження 1 робимо висновок, що у випадку $s \leq m+1 / 2$ виконується включення

$$
P_{0}(\eta(f, g)) \in \mathcal{H}_{\lambda, s, \varphi}(\Omega, \Gamma) \cap(A, B)\left(H_{A, \lambda}^{\sigma}(\Omega)\right)=(A, B)\left(H_{A, \lambda}^{s, \varphi}(\Omega)\right),
$$


а у випадку $s>m+1 / 2-$ включення

$$
P_{0}(\eta(f, g)) \in \mathcal{H}_{s-2 q, s, \varphi}(\Omega, \Gamma) \cap(A, B)\left(H_{A, \lambda}^{\sigma}(\Omega)\right)=(A, B)\left(H^{s, \varphi}(\Omega)\right) .
$$

Тому $P_{0}(\eta(f, g))=(A, B) u_{1}$ для деякого $u_{1} \in H^{s, \varphi}(\Omega) \cap H_{A, \lambda}^{\sigma}(\Omega)$. Тоді

$$
\eta(f, g)=P_{0}(\eta(f, g))+\left(I-P_{0}\right)(\eta(f, g))=(A, B) u_{1}+u_{1}^{\circ},
$$

де $I$ - тотожній оператор, а $u_{1}^{\circ}:=\left(I-P_{0}\right)(\eta(f, g)) \in N_{0}$. Kрім того, за умовою, $(A, B) u=$ $(f, g)$. Звідси отримуємо, що

$$
(A, B)\left(u-u_{1}\right)=(1-\eta)(f, g)+u_{1}^{\circ} .
$$

Оскільки $1-\eta=0$ на $V$ і $u_{1}^{\circ} \in N_{0} \subset C^{\infty}(\bar{\Omega}) \times\left(C^{\infty}(\Gamma)\right)^{q}$, a supp $\chi \subset V$, то за теоремою про локальне підвищення регулярності розв'язків еліптичних крайових задач у просторах Соболєва-Ройтберга [4] (теорема 7.2.1) виконується включення

$$
w:=\chi\left(u-u_{1}\right) \in \bigcap_{\theta \geq r} H^{\theta,(r)}(\Omega)=C^{\infty}(\bar{\Omega}) .
$$

Таким чином, $\chi u=\chi u_{1}+w \in H^{s, \varphi}(\Omega)$, бо $u_{1} \in H^{s, \varphi}(\Omega)$. Отже, $u \in H_{\mathrm{loc}}^{s, \varphi}\left(\Omega_{0}, \Gamma_{0}\right)$ з огляду на довільність вибору функції $\chi$.

Теорему 2 доведено.

\section{Література}

[1] Вентиель А. Д. О граничных условиях для многомерных диффузионных процессов // Теория вероятн. и ее примен. - 1959. - 4. - Р. 172 - 185. (Переклад англійською: Ventcel' $A$. D. On boundary conditions for multi-dimensional diffusion processes // Theory Probab. Appl. - 1959. - 4. - P. 164 - 177.)

[2] Красильников В. Н. О решении некоторых гранично-контактных задач линейной гидродинамики // Прикл. мат. мех. - 1961. - 25, № 4. - С. 764 - 768. (Переклад англійською: Krasil'nikov $V$. N. On the solution of some boundary-contact problems of linear hydrodynamics // J. Appl. Math. Mech. - 1961. - 25, № 4. - P. 1134 - 1141.)

[3] Вешев В.А., Коузов Д.П. О влиянии среды на колебания пластин, сочлененных под прямым углом // Акустический журнал. - 1977. - 23, № 3. - С. 368 - 377. (Переклад англійською: Veshev V. A., Kouzov D. P. Influence of the medium on the vibrations of plates joined at right angles // Acoustical Physics. - 1977. - 23, № 3. - P. $206-211$.

[4] Roitberg Ya. A. Elliptic boundary value problems in the spaces of distributions. Dordrecht: Kluwer Acad. Publisher, 1996. - xii+415 p.

[5] Kozlov V. A., Maz'ya V. G., Rossmann J. Elliptic boundary value problems in domains with point singularities. - Providence: Amer. Math. Soc., 1997. - 414 p.

[6] Hörmander L. Linear partial differential operators. - Berlin: Springer, 1963. - 285 p. (Переклад російською: Хермандер Л. Линейные дифференциальные операторы с частными производными. - Москва: Мир, 1965. - 380 с.) 
[7] Hörmander L. The analysis of linear partial differential operators. II: Differential operators with constant coefficients.- Berlin: Springer, 1983. - viii +391 р. (Переклад російською: Хермандер Л. Анализ линейных дифференциальных операторов с частными производными. Т. 2. - Москва: Мир, 1986. - 456 с.)

[8] Jacob N. Pseudodifferential operators and Markov processes: In 3 volumes. - London: Imperial College Press, 2001, 2002, 2005. - xxii +493 p., xxii +453 p., xxviii +474 p.

[9] Mikhailets V. A., Murach A. A. Hörmander spaces, interpolation, and elliptic problems. - Berlin, Boston: De Gruyter, 2014. - xii+297 р. (Видання російською доступне як arXiv:1106.3214.)

[10] Nicola F., Rodino L. Global Pseudodifferential Calculas on Euclidean spaces. - Basel: Birkhäser, 2010. $-\mathrm{x}+306 \mathrm{p}$.

[11] Paneah B. The oblique derivative problem. The Poincaré problem.- Berlin: Wiley-VCH, 2000. - 348 p.

[12] Stepanets A. I. Methods of approximation theory. - Utrecht: VSP, 2005.

[13] Triebel H. The structure of functions. - Basel: Birkhäser, 2001. - xii +425 p.

[14] Mikhailets V. A., Murach A. A. Elliptic operators in a refined scale of functional spaces // Ukrainian Math. J. - 2005. - 57, № 5. - P. 817 - 825.

[15] Mikhailets V. A., Murach A. A. Refined scales of spaces and elliptic boundary-value problems. II // Ukrainian Math. J. - 2006. - 58, № 3. - P. 398 - 417.

[16] Mikhailets V. A., Murach A. A. Regular elliptic boundary-value problem for homogeneous equation in two-sided refined scale of spaces // Ukrainian Math. J. 2006. - 58, № 11. - P. 1748 - 1767.

[17] Mikhailets V. A., Murach A. A. Elliptic operator with homogeneous regular boundary conditions in two-sided refined scale of spaces // Ukr. Math. Bull. -2006 . - 3, № 4. P. $529-560$.

[18] Mikhailets V. A., Murach A. A. Refined scales of spaces and elliptic boundary-value problems. III // Ukrainian Math. J. - 2007. - 59, № 5. - P. $744-765$.

[19] Mikhailets V. A., Murach A. A. An elliptic boundary-value problem in a two-sided refined scale of spaces. - Ukrainian Math. J. - 2008. - 60, № 4. - P. $574-597$.

[20] Mikhailets V. A., Murach A. A. The refined Sobolev scale, interpolation, and elliptic problems // Banach J. Math. Anal. - 2012. - 6, № 2. - P. 211 - 281.

[21] Anop A. V., Murach A. A. Parameter-elliptic problems and interpolation with a function parameter // Methods Funct. Anal. Topology. - 2014. - 20, No 2. - P. 103-116.

[22] Anop A. V., Murach A. A. Regular elliptic boundary-value problems in the extended Sobolev scale // Ukrainian Math. J. - 2014. - 66, № 7. - P. 969 - 985.

[23] Anop A. V., Kasirenko T. M. Elliptic boundary-value problems in Hörmander spaces // Methods Funct. Anal. Topology. - 2016. - 22, № 4. - P. 295 - 310. 
[24] Mikhailets V. A., Murach A. A. Extended Sobolev scale and elliptic operators // Ukrainian Math. J. - 2013. - 65, № 3. - P. 435 - 447.

[25] Mikhailets V. A., Murach A. A. Interpolation Hilbert spaces between Sobolev spaces // Results Math. - 2015. - 67, № 1. - P. $135-152$.

[26] Denk R., Faierman M. An elliptic boundary problem acting on generalized Sobolev spaces // arXiv:1710.01959v1. - 2017. - 21 p.

[27] Касіренко Т. М., Мурач О. О. Еліптичні задачі з крайовими умовами високих порядків у просторах Хермандера // Укр. мат. журн. - 2017. - 69, № 11. - С. 1486 1504.

[28] Lions J.-L., Magenes E. Problèmes aux limites non homogénes, V// Ann. Scuola Norm. Sup. Pisa (3). - 1962. - 16. - P. 1 - 44. (Italian)

[29] Lions J.-L., Magenes E. Problèmes aux limites non homogénes, VI // J. Anal. Math.-1963. - 11. - P. $165-188$.

[30] Лионс Ж.-Л., Мадженес Э. Неоднородные граничные задачи и их приложения. Москва: Мир, 1971. - 372 с.

[31] Ройтберг Я. А. Теоремы о гомеоморфизмах, осуществляемых эллиптическими операторами // Доклады АН СССР. - 1968. - 180, № 3. - С. 542-545. (Переклад англійською: Roitberg Ja. A. Theorems on homeomorphisms which can be realized by elliptic operators // Dokl. Math. - 1968. - 9. - P. 656-660).

[32] Костарчук Ю.В., Ройтберг Я.А. Теореми про ізоморфізми для еліптичних граничних задач з граничними умовами, які не є нормальними // Укр. мат. журн. 1973. - 25, № 2. - С. 271 - 277. (Переклад англійською: Kostarchuk Ju.(Yu.) V., Roitberg Ja.(Ya.) A. Isomorphism theorems for elliptic boundary value problems with boundary conditions that are not normal // Ukrainian Math. J. - 1973. - 25, № 2. - P. $222-226$.)

[33] Murach A. A. Extension of some Lions-Magenes theorems // Methods Funct. Anal. Topology. -2009 . - 15, № 2. - P. $152-167$.

[34] Михайлеи B. А., Мурач A. А. Индивидуальные теоремы о разрешимости эллиптических за-дач и пространства Хермандера // Доповіді НАН України. - 2011. - № 4. - C. $30-36$.

[35] Murach A. A., Chepurukhina I. S. Elliptic boundary-value problems in the sense of Lawruk on Sobolev and Hörmander spaces // Ukrainian Math. J. - 2015. - 67, № 5. P. $764-784$.

[36] Agranovich M. S. Elliptic boundary problems // Encycl. Math. Sci. Vol. 79. Partial differential equations, IX. - Berlin: Springer, 1997. - P. 1 - 144.

[37] Функииональный анализ / Под общ. ред. С. Г. Крейна. - Москва: Наука, 1972. $544 \mathrm{c}$.

[38] Seneta E. Regularly varying functions. - Berlin: Springer, 1976. - 112 р. (Переклад російською: Сенета Е. Правильно меняющиеся функции. - М.: Наука, 1985. - 144 с.) 
[39] Bingham N. H., Goldie C. M., Teugels J. L. Regular variation. - Cambridge: Cambridge Univ. Press, 1989. - 512 p.

[40] Волевич Л. Р., Панеях Б. П. Некоторые пространства обобщенных функций и теоремы вложения // Успехи мат. наук. - 1965. - 20, № 1. - С. 3 - 74. (Переклад англійською: Volevich L. R., Paneah B. P. Certain spaces of generalized functions and embedding theorems // Russian Math. Surveys. - 1965. - 20, № 1. - P. 1 - 73.)

[41] Ройтберг Я. А. Эллиптические задачи с неоднородными граничными условиями и локальное повышение гладкости вплоть до границы обобщенных решений // Доклады АН СССР. - 1964. - 157, № 4. - С. 798 - 801.

[42] Гохберг И. Ц., Крейн М. Г. Основные положения о дефектных числах, корневых векторах и индексах линейных операторов // Успехи матем. наук. - 1957. - 12, № 2. - С. 43 - 118. (Переклад англійською: Gohberg I. C., Krein M. G. The basic propositions on defect numbers, root numbers, and indices of linear operators // Amer. Math. Soc. Transl., Ser. 2. - 1960. - 13. - P. 185 - 264.)

[43] Mikhailets V. A., Murach A. A. Interpolation with a function parameter and refined scale of spaces // Methods Funct. Anal. Topology. - 2008. - 14, № 1. - P. $81-100$. 